\title{
Respiratory complications during initial rehabilitation and survival following spinal cord injury in Sweden: a retrospective study
}

\author{
Charlotta Josefson $\mathbb{1}^{1}{ }^{1} \cdot$ Tiina Rekand $\mathbb{1}^{1,2} \cdot$ Åsa Lundgren-Nilsson ${ }^{1} \cdot$ Katharina S. Sunnerhagen ${ }^{1}$
}

Received: 12 May 2020 / Revised: 26 August 2020 / Accepted: 1 September 2020 / Published online: 22 September 2020

(c) The Author(s), under exclusive licence to International Spinal Cord Society 2020

\begin{abstract}
Study design Retrospective study.

Objectives To determine prevalence of respiratory complications in individuals with spinal cord injury (SCI) during the initial rehabilitation at the spinal cord injury unit (SCU) and to describe the subsequent effect on mortality.

Setting The SCU at the university hospital in Gothenburg, Sweden.

Methods We reviewed the medical charts of newly injured persons with SCI who were admitted to the SCU between 1/1/ 2010 and 12/31/2014. Outcome measures were time to death, length of stay, occurrence of respiratory complications, and the use of breathing aids.

Results A total of 136 consecutive individuals were included; 53\% with cervical SCI and 20\% with lower SCI suffered from one or several respiratory complications during their initial rehabilitation in the SCU. At follow-up, 10/1/2018, 20\% of the individuals were deceased. The most common cause of death was related to respiratory insufficiency. The individuals with respiratory complications during the initial rehabilitation in the SCU had particularly shortened survival compared with those without. The relative risk (RR) of dying if the person suffered from any respiratory complications during their initial rehabilitation in the SCU was 2.1 times higher than for those with no respiratory complications (RR, 2.1; 95\% CI, 1.1-3.9). Conclusions Having respiratory complications at the SCU provides preliminary data to support the claim that respiratory complications predict premature mortality. Early diagnosis and prophylactic measures seem to be necessary to mitigate the adverse consequences of serious respiratory problems.
\end{abstract}

\section{Introduction}

Traumatic injury to the cervical and upper thoracic spinal cord is associated with variable degrees of pulmonary dysfunction and disability depending on the level and completeness of the injury [1-3]. Respiratory impairment after spinal cord injury ( $\mathrm{SCI}$ ) causes complications and is equally as frequent in lower cervical (C5-C8) and thoracic T1-T12 injuries [1]. Overall, the incidence of respiratory complications in the acute phase is up to $80 \%$ [4-6].

Charlotta Josefson

charlotta.josefson@gu.se

1 Department of Clinical Neuroscience, Sahlgrenska Academy, Institute of Neuroscience and Physiology, Gothenburg University, Gothenburg, Sweden

2 Department of Neurology, Haukeland University Hospital, Bergen, Norway
Clinically, it is not always easy to diagnose respiratory complications, as they may be masked by other SCI-related features. Respiratory complications associated with SCI are the most common cause of morbidity and mortality in both the acute phase and long-term [2,7-11], followed by circulatory causes, neoplasms, and urogenital problems for individuals surviving the first year after a traumatic SCI [12]. Survival during the first year after SCI has improved since the 1970s [11], but long-term survival has not changed [9-11].

Previous studies have shown that respiratory complications influence mortality after SCI $[2,13]$. Pneumonia is the most common cause of death in individuals with SCI in the chronic phase [14]. However, information regarding the influence of modern rehabilitation, acute respiratory complications, and length of survival after SCI is scarce. Therefore, we studied respiratory complications at the spinal cord injury unit (SCU) and followed-up overtime to support the claim that respiratory complications predict premature mortality. 


\section{Methods}

This retrospective study utilized data retrieved from medical charts and the official cause of death registry at the Swedish National Board of Health and Welfare. The study was carried out at the SCU at Sahlgrenska University Hospital (SU) in Gothenburg, Sweden. The SCU (at that time) provided the initial care and rehabilitation after SCI in the Gothenburg area (population around 800,000) and all traumatic and most non-traumatic came to the SCU. We did not admit malignant causes nor cauda equina.

We performed consecutive inclusion of all newly injured persons with SCI caused by traumatic or non-traumatic events who were admitted to the SCU at SU between 1/1/ 2010 and 12/31/2014. A total of 136 individuals were included in the study. Follow-up regarding mortality ended 10/1/2018. Individuals not permanently living in Sweden, who were transferred to other departments, deceased within 2 days after admission, or did not have a newly diagnosed SCI were excluded (Fig. 1).

The inpatient electronic medical records were reviewed to collect the following data from the individuals' initial care and rehabilitation in the SCU: American Spinal Injury Association Impairment Scale (AIS) at discharge [15], standard data according to the international SCI core set $[16,17]$, and Charlson Comorbidity Index (CCI) calculated for the person at the time of injury. The need for a breathing aid was defined as the use of noninvasive ventilation (NIV), continuous positive airway pressure (CPAP) or bi-level positive airway pressure (BiPAP); tracheostomy and the use of an invasive ventilator; the use of a cough assist machine; or requiring supplementary oxygen for at least $8 \mathrm{~h}$ a day that persisted at discharge. Individuals who required any of these breathing aids during surgery and were then weaned off prior to admission to the SCU were not included or analyzed, nor were any respiratory complications that emerged prior to admittance to the SCU. However, if any of those persons experienced any respiratory complications again once they had been admitted to the SCU, they were indeed included in the study. Respiratory complications extracted from the medical records were defined as pneumonia, severe mucus plugs, or transfer to the intensive care unit (ICU) due to respiratory failure. The diagnosis of pneumonia had to be stated in the medical record as a clinical diagnosis with the International Classification of Diseases (ICD) code, in addition to treatment with appropriate antibiotics.

Date and cause of death defined by the ICD codes were retrieved from the official cause of death registry at the Swedish National Board of Health and Welfare.

Length of stay (LOS) for initial care and rehabilitation in the SCU was calculated in days from the time of admittance to the SCU until the time of discharge from the SCU. LOS
153 patients

January 2010- December 2014

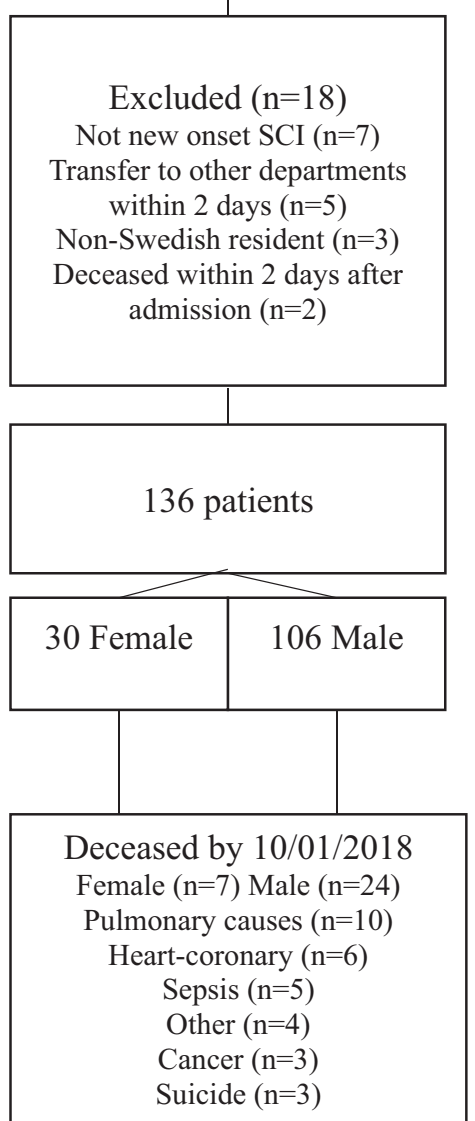

Fig. 1 Flowchart of included persons. Number and reason for exclusion of persons and number of persons deceased and reson to death.

was dichotomized; long LOS was defined as a duration greater than the median LOS for the whole studied sample. The median was chosen instead of mean because the LOS appeared to follow a skewed distribution. Age at onset was also dichotomized, young was defined as 51 years and younger and old was defined as an age above. Fifty-one years being the median age at onset, median was chosen instead of mean since the age at onset appeared to follow a skewed distribution.

Statistical analyses were performed using the IBM Statistical Package for Social Sciences (SPSS) version 24 for Windows (SPSS Inc., Chicago, IL, USA). The results are presented using quantitative descriptive statistics with mean, median, standard deviation, minimum and maximum, frequency, and proportion (\%) when appropriate. Fisher's exact test was used for categorical data. Relative risk (RR) was used to compare the risk of outcome (LOS and survival) between groups when appropriate, and a 95\% confidence interval (CI) not covering 1 was considered 
significant. A Kaplan-Meier survival curve was used to investigate the relationship between survival and respiratory complications in the SCU. As well as a Cox regression model was used to investigate the relationship between survival and respiratory complications in the SCU, age and level of injury (cervical or other). $P \leq 0.05$ was considered significant.

\section{Results}

\section{Sample characteristics}

A total of 153 people were identified, 136 of whom met the inclusion criteria (Fig. 1). The individuals had SCI caused by traumatic $(84 \%, n=114)$ or non-traumatic events. The most common traumatic event was fall $(33 \%, n=45)$, and the most common non-traumatic event was infection $(5 \%$, $n=7)$ or vascular events $(5 \%, n=7)$. The median age at onset of SCI was 51 years (IQR: 33-65 years); four people were $<18$ years old at the time of SCI. Twenty-two percent of the included individuals were female.

The mean CCI was 1 (IQR: $0-3)$. Cervical SCIs $(n=83)$ were more common than thoracic-lumbar SCIs $(n=53)$. Details of the demographics are presented in Table 1.

The median LOS in the SCU was 65 days (IQR 41-118 days).

\section{Need for breathing aid during initial rehabilitation in the SCU}

A total of $38 \%(n=52)$ of the study population required some breathing aid during their initial rehabilitation period in the SCU. NIV was used by $19 \%(n=26)$ of the sample,

Table 1 Baseline demographics of the included individuals.

\begin{tabular}{lclll}
\hline Grouping variables & $\begin{array}{l}\text { Total sample } \\
(n=136)\end{array}$ & $\begin{array}{l}\text { Alive } \\
(n=105)\end{array}$ & $\begin{array}{l}\text { Dead } \\
(n=31)\end{array}$ & $P$ value \\
\hline $\begin{array}{l}\text { Spinal cord injury level } \\
\text { Cervical }\end{array}$ & $83(61.0)$ & $62(74.7)$ & $21(25.3)$ & $0.411^{\mathrm{a}}$ \\
$\quad$ Thoracic-sacral & $53(39.0)$ & $43(81.1)$ & $10(18.9)$ & \\
SCI standard core set, level and severity & & & \\
C1-4 AIS ABC & $22(16.9)$ & $16(72.7)$ & $6(27.3)$ & $0.237^{\mathrm{b}}$ \\
C5-C8 AIS ABC & $23(17.7)$ & $18(78.3)$ & $5(21.7)$ & \\
T1-S5 AIS ABC & $34(26.2)$ & $27(79.4)$ & $7(20.6)$ & \\
$\begin{array}{l}\text { All levels AIS D } \\
\text { Ventilator-dependent at }\end{array}$ & $74(33.8)$ & $36(81.8)$ & $8(18.2)$ & \\
discharge, all levels & $7(5.4)$ & $3(42.9)$ & $4(57.1)$ & \\
\hline
\end{tabular}

Data are given as $n(\%)$.

${ }^{\text {a}}$ Fisher's exact test.

${ }^{b}$ Pearson chi-squared.

${ }^{\mathrm{c}}$ The AIS classification was unavailable for 6 patients, total 130 patients. and $32 \%(n=44)$ had a tracheostomy and required invasive ventilation at some point during their stay at the SCU.

A cough assist machine was used by $26 \%(n=35)$ at some point during their stay in the SCU. For $7 \%(n=9)$ of individuals, the need for supplementary oxygen at least $8 \mathrm{~h}$ a day persisted at discharge and $5 \%(n=7)$ were ventilatordependent at discharge. More than half of the individuals $(n=43)$ with a cervical SCI needed a breathing aid during their initial rehabilitation in the SCU, and $17 \%(n=9)$ of the individuals with lower injuries required the same.

\section{Respiratory complications during initial rehabilitation in the SCU}

A total of $40 \%(n=54)$ of individuals had acute respiratory complications during their stay in the SCU. Pneumonia was diagnosed in $35 \%(n=48)$ of cases; median being diagnosed with pneumonia two times while at the SCU (IQR: 1-2 times). Removal of the mucus plugs was needed in $27 \%$ $(n=37)$ of individuals. Nine people $(7 \%)$ were transferred from the SCU to the ICU due to respiratory failure. More than half of the individuals with cervical SCI $(n=43)$ had respiratory complications during their initial rehabilitation in the SCU, and $20 \%(n=11)$ of individuals with lower injuries experienced the same.

\section{LOS and correlation with the need for a breathing aid and/or respiratory complications during the initial rehabilitation in the SCU}

Those who required breathing aids as defined above during their initial rehabilitation in the SCU had a $57 \%$ increase in the risk of a LOS $>65$ days compared to individuals who did not require any kind of breathing aid (RR, 1.57; 95\% CI, 1.14-2.16). The median LOS was 65 days and was chosen as the cut off when LOS was dichotomized.

The individuals who suffered from any respiratory complications (pneumonia, obstruction by mucus plugs, or transfer to the ICU due to respiratory failure) during their initial rehabilitation in the SCU had a $76 \%$ increase in the risk of a LOS $>65$ days (RR, 1.76; 95\% CI, 1.3-2.4; $p<$ 0.005) Fig. 2.

\section{Respiratory complications during initial rehabilitation in the SCU and survival}

Of all people included in the study, $23 \%(n=31)$ are now deceased. Follow-up regarding mortality ended 2018-01-10. Median time to follow-up was 6 years (IQR: 5-8 years) Fig. 3. Respiratory causes contributed to one-third of the deaths $(n=10)$, followed by heart/coronary $(n=6)$ and sepsis and other infections $(n=5)$. One-third $(n=7)$ of the deceased individuals with cervical SCI and three individuals 


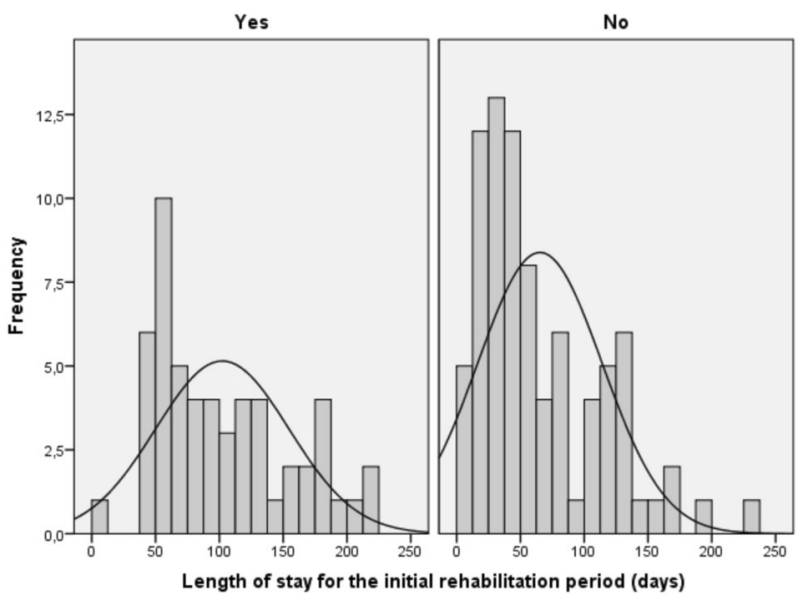

Fig. 2 Barchart over number of days spent in the SCU. Length of stay at the SCU and respiratory complications yes/no during the initial rehabilitation period, $\mathrm{y}$-axis number of persons, $\mathrm{x}$-axis number of days.

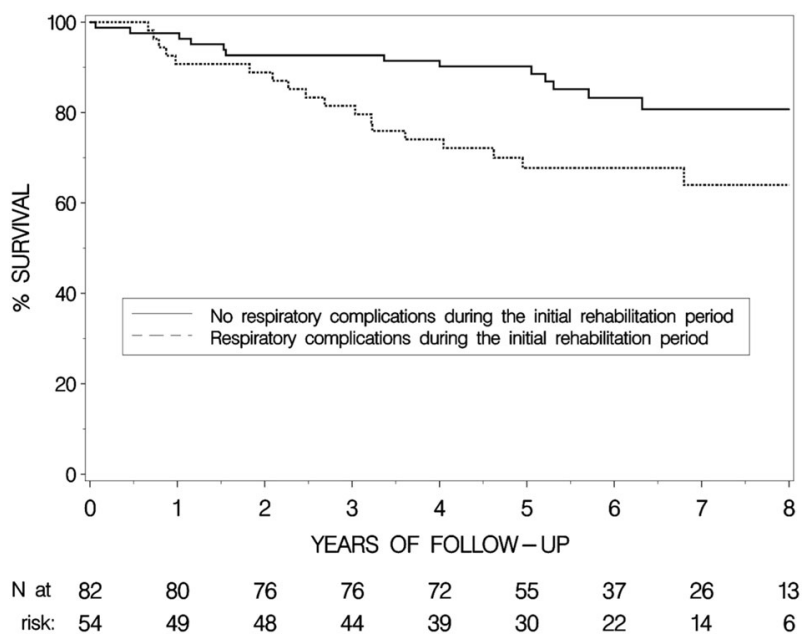

Fig. 3 Kaplan-Meier survival curve. Time to follow-up regarding mortality ended 10/1/2018. Table with numbers at risk, the top row represents those who experienced no respiratory complications at the SCU, the bottom row represents those with respiratory complications at the SCU.

with a lower SCI (out of 10,30\%) died from respiratory causes. The median age for the deceased was 72 years (IQR: 57-81 years). The median survival after SCI onset was 2 years and 8 months (IQR: 53-241 weeks).

The RR of dying if the person suffered from any respiratory complications during their initial rehabilitation in the SCU was 2.1 times higher than for those with no respiratory complications (RR, 2.10; 95\% CI, 1.1-3.9). While a history of pneumonia was associated with $72 \%$ higher mortality, this was not statistically significant (RR, $1.72 ; 95 \%$ CI, 0.9-3.2).

When using a Cox regression analysis, a history of respiratory complications in the SCU was associated with a higher mortality and a tendency of a shorter life span, this was not statistically significant, as the CI covered 1 (HR, 1.88 ; 95\% CI, 0.9-4.1; p 0.11). In the Cox regression analysis the variable that was statistically significant was age where the older individuals had a higher risk of a shorter life span (HR, 3.64; 95\% CI, 1.6-8.5; $p<0.01)$. A cervical injury was associated with a higher mortality and a tendency of a shorter life span, this was not statistically significant, as the CI covered 1 (HR 1.15; 95\% CI, 0.5-2.6; p 0.73).

\section{Death due to respiratory causes}

Ten people died from respiratory causes, including eight who suffered from pneumonia during their initial rehabilitation in the SCU and had a 4.3 times higher risk (RR, 4.27; 95\% CI, 1.1-16.9) of dying from respiratory causes later compared to those who did not suffer from pneumonia at the SCU. Six out the ten individuals required use of the cough assist machine during their stay in the SCU, which also indicated a significantly higher risk of death due to respiratory causes (RR, 3.15; 95\% CI, 1.1-8.7).

\section{Discussion}

This study indicates an association between all acute respiratory complications in the SCU and survival. We also found an association between pneumonia in the SCU that may indicate association with death due to respiratory causes later on in life. The findings indicates the need for immediate diagnosis and treatment, as well as awareness of the risk respiratory complications may cause and interventions aiming to prevent these. The association between the early respiratory complications and survival means that the optimal treatment may influence the long-term outcome. Even though the Cox regression analysis was not statistically significant for respiratory complications it did show a tendency of a shorter life span if the person had suffered from respiratory complications in the SCU.

Acute respiratory complications were present in all levels of SCI in our study. Cervical SCIs are more prone to respiratory complications [1,2], but our results emphasize that acute respiratory complications may occur at all levels of SCI and should be managed both acutely and at followup. The occurrence of respiratory complications and use of breathing aids is exceptionally high during the first weeks following a SCI [1]. Our results that respiratory complications significantly increase the risk of mortality in less severely injured persons are supported by previous findings [18]. Nor did we find evidence in our study to claim that the level of SCI significantly influenced survival [19].

Our study provides preliminary data that having pneumonia and other respiratory complications at the SCU support the claim that respiratory complications predict 
premature mortality. Early diagnosis and prophylactic measures are necessary to mitigate the adverse consequences of serious respiratory problems. People who are diagnosed with pneumonia in the SCU require follow-up after discharge. Physical examination in follow-up and patient history alone do not necessarily provide sufficient information about respiratory status why for example spirometry and chest X-ray could be useful.

We showed that individuals with pneumonia during their initial rehabilitation likely die later due to respiratory causes. Therefore, that pneumonia that occurs during acute rehabilitation may indicate respiratory dysfunction and predict shortened survival.

Larger studies are needed to analyze assistive breathing and respiratory dysfunction separately. We found that the use of breathing aids influences LOS. Such use may be an indicator of respiratory dysfunction; thus, the length of initial rehabilitation and follow-up of such complications should be clearly defined. Winslow et al. [20] showed that respiratory complications experienced during the initial hospitalization have a greater impact on LOS and hospital cost than the level of injury itself. Our study demonstrates that the respiratory complications may indicate association with longer LOS regardless of the level of the SCI. The median survival was 2 years and 8 months, which is consistent with previous studies reporting high mortality due to various causes in the first 2 years after a SCI [11].

\section{Clinical relevance}

Respiratory complications during initial rehabilitation should be considered serious, as they have a life-long impact on the individual. All persons with SCI and respiratory complications require a long initial rehabilitation in the SCU and should be thoroughly followed-up by respiratory disease specialists after discharge. Anything that can reduce respiratory complications both during the initial rehabilitation and after discharge might reduce complications including mortality and morbidity, LOS, and costs.

\section{Study limitations}

The study has a retrospective design, and missing data in the medical records cannot be restored. We do not know if all respiratory complications were properly documented in the medical charts; for example, could mucus plugs be under-reported? For 6 of the 136 individuals included in the study, the AIS score was missing from their medical chart. Some subgroups were small, and as such the percentages may be misleading. Due to small subgroups, the data for the RR calculations were unadjusted. The same reason, the different subgroups being too small, is why the study is based mainly on univariate analyzes. These type of analyzes can help us predict outcomes but are not as precise in determine causation as multivariate analyzes are. Hence caution is of importance when interpreting the data. However, the present study provides important insights into the course of respiratory complications in all levels and types of SCI caused by traumatic or non-traumatic events.

\section{Conclusion}

Persons with a cervical SCI have a significantly higher risk of respiratory complications. However, $20 \%$ of individuals with lower injuries may also suffer from respiratory complications and may require breathing aids. Respiratory complications may lead to longer rehabilitation and shorter survival regardless of the level and completeness of the SCI. Therefore, early diagnosis and prophylactic measures seem to be necessary to mitigate the adverse consequences of serious respiratory problems.

\section{Data availability}

The datasets analysed during the present study are not publicly available due to Swedish regulation (https:// etikprovningsmyndigheten.se/for-forskare/vad-sager-lagen/) that state that data cannot be made available for more than what has been approved by the Ethical Review Board. The datasets generated and/or analyzed during the current study are available from the corresponding author on reasonable request.

Funding This research was supported by a grant from the Sahlgrenska University Hospital Foundation and Hjalmar Svensson Foundation. The study was financed by grants from the Swedish state under the agreement between the Swedish government and County Councils, ALF agreement 71980 (KSS).

Author contributions CJ collected data, conducted the statistical analysis with assistance from statistician Thomas Karlsson, and was responsible for writing the paper. TR, ÅLN, and KSS contributed to the study design and the writing and editing of the paper. All contributors have read and approved the final version of the paper.

\section{Compliance with ethical standards}

Conflict of interest The authors declare that they have no conflict of interest.

Ethical approval The regional ethical review board in Gothenburg gave ethical approval (reference numbers 383-17 and T560-18) according to the guidelines of the Declaration of Helsinki. Clinical data collected during the inclusion period did not require informed consent according to Swedish law on personal data (The Personal Data Act, Swedish law No. SFS 1998:2014).

Publisher's note Springer Nature remains neutral with regard to jurisdictional claims in published maps and institutional affiliations. 


\section{References}

1. Jackson $\mathrm{AB}$, Groomes TE. Incidence of respiratory complications following spinal cord injury. Arch Phys Med Rehabil. 1994;75:270-5.

2. Schilero GJ, Bauman WA, Radulovic M. Traumatic spinal cord injury: pulmonary physiologic principles and management. Clin Chest Med. 2018;39:411-25.

3. Berlowitz DJ, Wadsworth B, Ross J. Respiratory problems and management in people with spinal cord injury. Breathe. 2016;12:328-40.

4. Fishburn MJ, Marino RJ, Ditunno JF Jr. Atelectasis and pneumonia in acute spinal cord injury. Arch Phys Med Rehabil. 1990;71:197-200.

5. Reines HD, Harris RC. Pulmonary complications of acute spinal cord injuries. Neurosurgery. 1987;21:193-6.

6. Tollefsen E, Fondenes O. Respiratory complications associated with spinal cord injury. Tidsskr Nor Laegeforen. 2012;132:1111-4.

7. Lemons VR, Wagner FC Jr. Respiratory complications after cervical spinal cord injury. Spine. 1994;19:2315-20.

8. Rabadi MH, Mayanna SK, Vincent AS. Predictors of mortality in veterans with traumatic spinal cord injury. Spinal Cord. 2013;51:784-8.

9. Shavelle RM, DeVivo MJ, Brooks JC, Strauss DJ, Paculdo DR. Improvements in long-term survival after spinal cord injury? Arch Phys Med Rehabil. 2015;96:645-51.

10. Shavelle RM, DeVivo MJ, Strauss DJ, Paculdo DR, Lammertse DP, Day SM. Long-term survival of persons ventilator dependent after spinal cord injury. J Spinal Cord Med. 2006;29:511-9.

11. Strauss DJ, Devivo MJ, Paculdo DR, Shavelle RM. Trends in life expectancy after spinal cord injury. Arch Phys Med Rehabil. 2006;87:1079-85.
12. Savic G, DeVivo MJ, Frankel HL, Jamous MA, Soni BM, Charlifue S. Causes of death after traumatic spinal cord injury-a 70-year British study. Spinal Cord. 2017;55:891-7.

13. Cao Y, Selassie AW, Krause JS. Risk of death after hospital discharge with traumatic spinal cord injury: a populationbased analysis, 1998-2009. Arch Phys Med Rehabil. 2013;94: 1054-61.

14. DeVivo MJ, Black KJ, Stover SL. Causes of death during the first 12 years after spinal cord injury. Arch Phys Med Rehabil. $1993 ; 74: 248-54$.

15. Roberts TT, Leonard GR, Cepela DJ. Classifications in brief: American Spinal Injury Association (ASIA) impairment scale. Clin Orthop Relat Res. 2017;475:1499-504.

16. Biering-Sorensen F, DeVivo MJ, Charlifue S, Chen Y, New PW, Noonan V, et al. International Spinal Cord Injury Core Data Set (version 2.0)-including standardization of reporting. Spinal Cord. 2017;55:759-64.

17. DeVivo MJ, Biering-Sorensen F, New P, Chen Y. Standardization of data analysis and reporting of results from the International Spinal Cord Injury Core Data Set. Spinal Cord. 2011;49: 596-9.

18. Cotton BA, Pryor JP, Chinwalla I, Wiebe DJ, Reilly PM, Schwab $\mathrm{CW}$. Respiratory complications and mortality risk associated with thoracic spine injury. J Trauma. 2005;59:1400-7.

19. Yang XX, Huang ZQ, Li ZH, Ren DF, Tang JG. Risk factors and the surgery affection of respiratory complication and its mortality after acute traumatic cervical spinal cord injury. Medicine. 2017;96:e7887.

20. Winslow C, Bode RK, Felton D, Chen D, Meyer PR Jr. Impact of respiratory complications on length of stay and hospital costs in acute cervical spine injury. Chest. 2002;121:1548-54. 